\title{
Digital response with femtosecond resolution in an optical AND gate
}

\author{
G.R. Collecutt *, P.D. Drummond \\ Department of Physics, University of Queensland, Brisbane, Qld 4072, Australia
}

Received 10 May 2000; received in revised form 14 July 2000; accepted 24 July 2000

\begin{abstract}
We present a numerical simulation of an all-optical AND logic gate utilising soliton formation in a planar waveguide with a parametric nonlinearity. We investigate switching performance as a function of input logic simultaneity and internal geometry. A highly digital response in the transmitted pulse energy is observed, with femtosecond time discrimination. (C) 2000 Elsevier Science B.V. All rights reserved.
\end{abstract}

For around three decades now, the all-optical processor has proved elusive, with mixed opinions as to how it may be achieved [1]. The growth and performance of silicon-based electronics has truly exceeded all expectations, but as a direct consequence of this performance, we have a rapidly growing global information network which is continually pushing networking technology to its limits. Fibre optics is very successfully solving the need for simple information propagation, but until recently, optical signals had to be continually received and re-sent for two purposes: amplification and routing. This process of receiving signals and re-sending them is expensive and slow in comparison to the huge bandwidth available with an op-

\footnotetext{
${ }^{*}$ Corresponding author. Tel.: +61-7-3365-3405; fax: +61-73365-1242.

E-mail address: collecug@physics.uq.edu.au (G.R. Collecutt).
}

tical fibre, and is one of the primary bottlenecks in an optical information network. Now that the erbium-doped fibre laser is proving to be a practical and effective optical amplifier, all that remains is to be able to route information while it remains in optical form. Signal routing, multiplexing, demultiplexing, and regeneration are the present applications for all-optical logic switching.

In this paper, we describe the theory of a parametric type II soliton-based optical AND gate. In particular, we find the switching performance to be a highly digital function of pulse timing. The optical AND gate can operate with a pulse FWHM as short as $30 \mathrm{fs}$, and it shows digital response with a resolution of order $1 \mathrm{fs}$, provided Raman scattering and higher-order dispersion can be neglected. A closely related type I device has already been experimentally demonstrated with 100 fs pulse duration [2].

To illustrate one of the main problems involved with all-optical switching, consider first the 
requirements for the operation of an AND logic gate. In order to obtain a logic true output, each of the logic inputs must know about the presence of the other. This is essentially a nonlinear process. However, light in the majority of dielectrics is the solution to the linear Maxwell equations, and thus does not interact with other light. A dielectric with nonlinear response is needed. In recent years, there have been considerable advances in the theory and manufacture of such nonlinear optical media, which now opens up a new and exciting range of possibilities for all-optical logic circuits. One such possibility is the implementation of an all-optical AND gate, as proposed by Drummond et al. [3] and reillustrated in Fig. 1.

In this method, two simultaneous dispersive input pulses are collided at the boundary of a planar parametric waveguide to form a $2+1 \mathrm{D}$ spatiotemporal soliton, which propagates towards the output port in a stable manner. If only one input pulse is present, then it simply disperses with little or no energy reaching the output. Clearly, if there are no pulses present, then there will be no output. Hence, AND gate logic is achieved. The basic entity used is the $2+1 \mathrm{D}$ parametric spatiotemporal soliton, which was proposed in 1997 by Malomed et al. [4], and was experimentally observed last year by Liu et al. [5]. The two main requirements are anomalous dispersion and (near) group velocity matching at the fundamental and second harmonic frequencies. We emphasise that

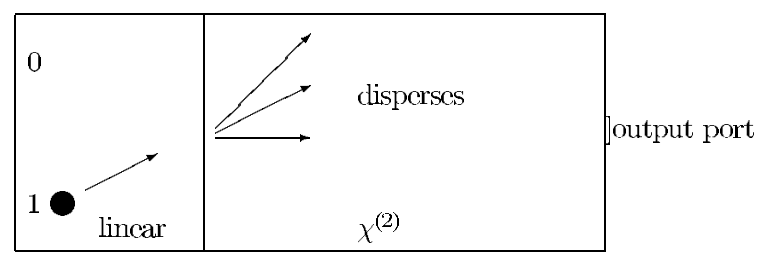

(a) Logic: $1 \& 0 \mapsto 0$

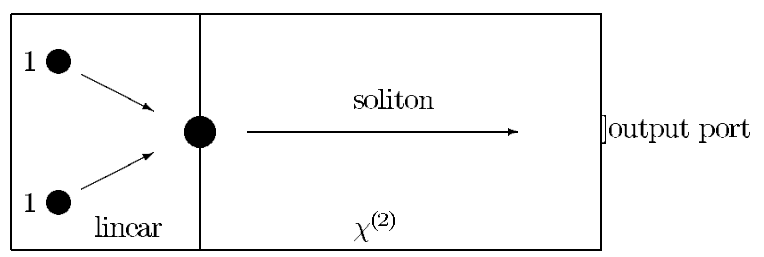

(b) Logic: $1 \& 1 \mapsto 1$

Fig. 1. Schematic of proposed AND gate implementation. the method uses nonlinear spatiotemporal soliton formation [6] as the key element. In this respect, it is completely distinct from other proposals [7-9] for optical logic gates based on soliton collisions or spatial soliton interactions.

There are two different methods by which the input pulses can interact: type I interaction, wherein the pulses are of the same polarisation; or type II interaction, wherein the pulses are of orthogonal polarisations. For the purpose of logic switching, it would be ideal for all input and output pulses to be identical, i.e., the same carrier frequency, polarisation, and similar shape and intensity. The difficulty with completely degenerate pulses, as pointed out by Assanto [9] and also by Drummond et al. [3], is that the interaction would be phase sensitive, and precise optical phasing is difficult to control. As a result, if we are to avoid controlling precise pulse phases, we must resort to making the pulses nondegenerate either in frequency or polarisation. Further, if we are to link serially a number of these optical AND gates, we need to convert one type of logic bit to another. This is more easily done in polarisation than in frequency, thus favouring type II interaction. Eqs. (1)-(3) describe the $2+1 \mathrm{D}$ nondegenerate (type II) parametric interaction in dimensionless coordinates [3]:

$$
\begin{aligned}
\frac{\partial \phi_{1}}{\partial \xi}=i[( & \frac{\partial^{2}}{\partial \tau^{2}}+\frac{\partial^{2}}{\partial \zeta^{2}}+i v_{\mathrm{GVM}} \frac{\partial}{\partial \tau} \\
& \left.+i \Gamma(\tau, \zeta)-1) \phi_{1}+\phi_{2}^{*} \phi_{3}\right]
\end{aligned}
$$

$$
\begin{aligned}
\frac{\partial \phi_{2}}{\partial \xi}=i[( & \frac{\partial^{2}}{\partial \tau^{2}}+\frac{\partial^{2}}{\partial \zeta^{2}}-i v_{\mathrm{GVM}} \frac{\partial}{\partial \tau} \\
& \left.+i \Gamma(\tau, \zeta)-1) \phi_{2}+\phi_{1}^{*} \phi_{3}\right]
\end{aligned}
$$

$$
\begin{gathered}
\frac{\partial \phi_{3}}{\partial \xi}=i\left[\left(\frac{1}{\sigma} \frac{\partial^{2}}{\partial \tau^{2}}+\frac{1}{2} \frac{\partial^{2}}{\partial \zeta^{2}}+i \Gamma(\tau, \zeta)-\gamma\right) \phi_{3}\right. \\
\left.+\phi_{1} \phi_{2}\right]
\end{gathered}
$$

where $\sigma$ is the ratio of dispersion between fundamental and second harmonic frequencies, and $\gamma$, a 
phase velocity mismatch parameter for the second harmonic field. For the results presented, $\sigma$ was set to 2 (yielding $\tau-\zeta$ symmetry) and $\gamma$ was set to 1 . The group velocities of the fundamental fields are assumed to symmetrically straddle the group velocity of the second harmonic field; the reciprocals of the velocities differing by $\pm v_{\mathrm{GVM}} . \Gamma(\tau, \zeta)$ is a damping field applied in the numerical simulations to absorb scattered radiation at the lattice boundaries. The theory here is somewhat idealised in that these equations neglect higher-order dispersion, Raman scattering, second harmonic group velocity mismatch, and spatial walk-off effects as well as any possible effects due to nonlinear dispersion. The variable transformation is given by

$$
\begin{aligned}
\phi_{i} & =\frac{\Phi_{i}}{\Phi_{0}}, \quad \xi=\frac{z}{z_{0}}, \quad \zeta=\frac{y}{y_{0}}, \\
\tau & =\frac{t-z / v_{\mathrm{g} 3}}{t_{0}}, \quad v_{\mathrm{GVM}}=\frac{z_{0}}{c t_{0}} \frac{n_{\mathrm{g} 2}-n_{\mathrm{g} 1}}{2},
\end{aligned}
$$

where $\left|\Phi_{0}\right|^{2}$ is a reference photon flux, $z$, the direction of propagation, $y$, the transverse coordinate, and $t$, the time. Eqs. (5) and (6) (derived from Ref. [11]) define the scalars $y_{0}, t_{0}$, and $\Phi_{0}$ :

$$
\begin{aligned}
& y_{0}=\sqrt{\frac{z_{0}}{2 k_{0}}}, \quad t_{0}=\sqrt{\frac{z_{0} \beta_{2}}{2}}, \quad \Phi_{0}=\frac{1}{\chi z_{0}}, \\
& \chi=\chi^{(2)} \sqrt{\frac{\hbar k_{0}^{3}}{\epsilon_{0} n^{3}}} \int u^{(1)}\left(u^{(2)}\right)^{*} u^{(1)} \mathrm{d} x,
\end{aligned}
$$

where $k_{0}$ is the fundamental wave number in free space, $\beta_{2}$, the second dispersion, $n$, the refractive index at the fundamental wavelength, and $\chi$, the parameter derived from the $\chi^{(2)}$ nonlinearity and mode shape integral.

To investigate this process of soliton formation, numerical simulations of type I and type II interactions have been performed using a central step partial difference integration method [10] on a 2D lattice. Figs. 2 and 3 display the results for type II interaction: Fig. 2 is for the case of only one input pulse, and Fig. 3, for the case of simultaneous input pulses. Here, we are plotting the transverse cross-section (through $\tau=0$ ) of total power density $\left(\left|\phi_{1}\right|^{2}+\left|\phi_{2}\right|^{2}+2\left|\phi_{3}\right|^{2}\right)$ as a function of propagation distance.
Due to reasons that will be discussed shortly, we require nonlinear evolution of the order of 10 units of $z_{0}$ in order to effect the switch. If this is required within an available length of $\chi^{(2)}$ media of say $50 \mathrm{~mm}$, then $z_{0}=5 \mathrm{~mm}$. Assuming (very approximately) a square mode profile, the mode shape integral may be evaluated as $1 / \sqrt{d}$, where $d$ is the depth of the planar guide. Taking typical values for nonlinearity, dispersion, and fundamental wavelength of $\chi^{(2)}=10 \mathrm{pm} / \mathrm{V}, \beta_{2}=25 \mathrm{ps}^{2} /$ $\mathrm{km}$, and $\lambda=1.5 \mu \mathrm{m}$, respectively, we obtain $y_{0}=$ $24.43 \mu \mathrm{m}$ and $t_{0}=7.91 \mathrm{fs}$. Finally, with a waveguide depth of $2 \mu \mathrm{m}$, we get $\Phi_{0}=1.58 \times 10^{12}$ (photons $/ \mathrm{ms})^{1 / 2}$.

Figs. 2 and 3 were originally obtained in dimensionless coordinates, in which the propagation was for 10 units of $z_{0}$ with the GVM between the fundamental fields set to zero. The initial pulses used were radially symmetric Gaussians $\left(r^{2}=\tau^{2}+\right.$ $\left.\zeta^{2}\right)$ with an amplitude of $\phi_{0}=7.5$ and radius $r_{0}=2$. Their initial transverse velocities were \pm 1 unit of $y_{0}$ for every unit of $z_{0}$, and for Fig. 3, their centres were still 0.40 units of $y_{0}$ apart and converging when they launched. The pulses were also simultaneous, i.e. both centred on $\tau=0$. The second harmonic field $\left(\phi_{3}\right)$ was initialised to zero. Using the above scaling, the input pulses were $98 \mu \mathrm{m}$ in width and $31.6 \mathrm{fs}$ in duration (FWHM, both cases). This yields a total energy for each input pulse of 22.6 pJ. Hence, for Fig. 3, the pulses were converging at an included angle of $0.56^{\circ}$, and their centres were still $9.8 \mu \mathrm{m}$ apart at the start of the nonlinear medium. The software package developed for these simulations is called XMDS, ${ }^{1}$ and the input scripts for the simulations depicted in Figs. 2 and 3 are available on the Internet. ${ }^{2}$

As can be seen in Fig. 3, a breathing soliton forms when both input pulses are present, whereas in Fig. 2, the input pulse energy of a single pulse is seen to disperse. It is reasonable to use either of the fundamental fields or even the second harmonic field for the switch output, but not all three. Here, we have chosen to plot total power density because it is the more revealing moment with regard to

\footnotetext{
${ }^{1} \mathrm{XMDS}$ is available at http://physics.uq.edu.au/xmds

${ }^{2} \mathrm{http} / / /$ physics.uq.edu.au/xmds/scripts
} 


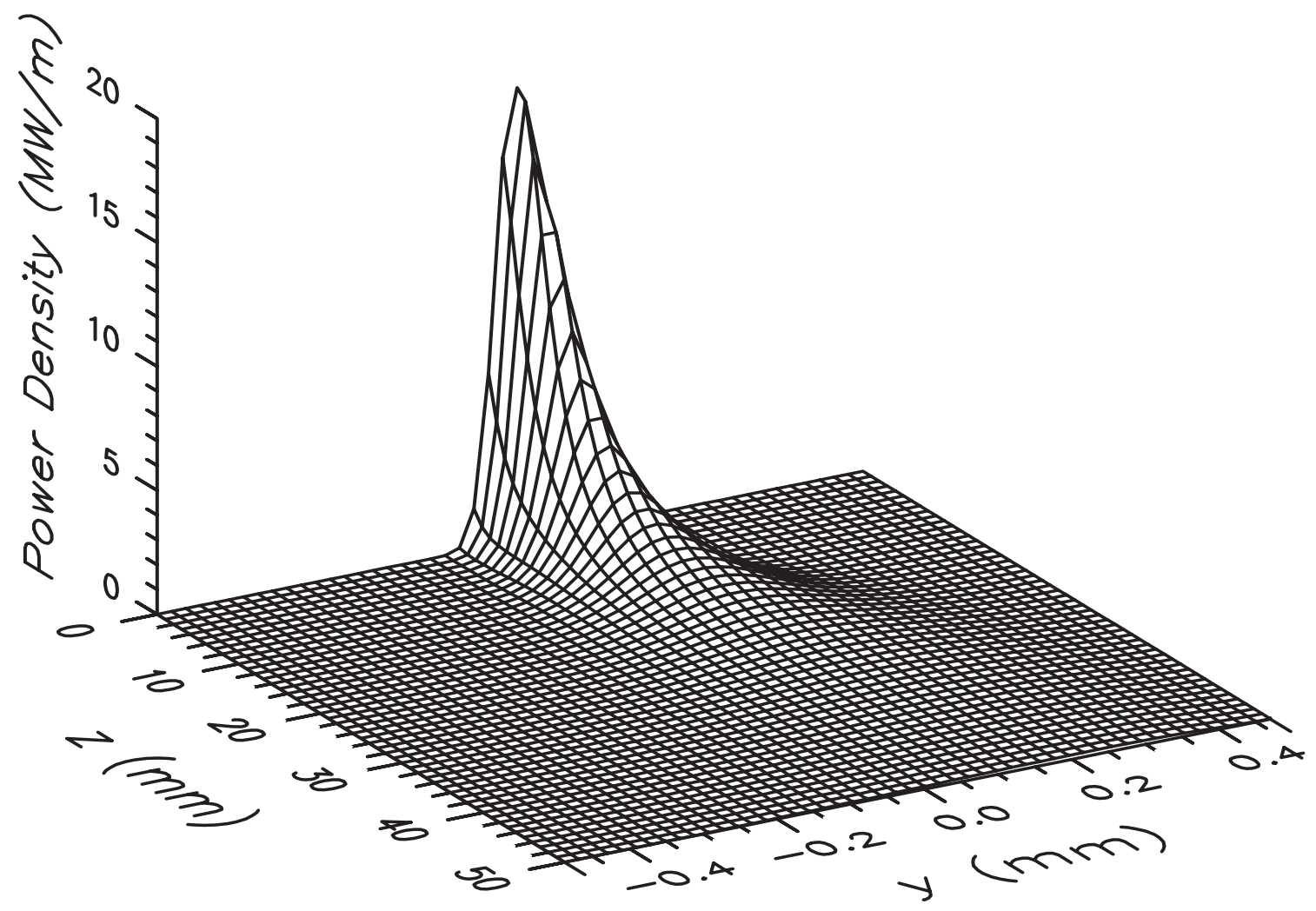

Fig. 2. Single pulse input.

understanding the dynamics of the interaction, not because it defines the output of the switch.

From our studies, we have found several interesting points of behaviour. Firstly, it is interesting to note that to form a soliton, the pulses must be overlapped as they enter the nonlinear medium. We have not been able to produce a soliton with input pulses that are initially separate.

Secondly, it is possible to repeat the above simulations for the case of completely degenerate pulses simply by superposing both inputs in one fundamental field and using the appropriate equations for frequency degenerate type I interaction. The pulses must be separate initially in order to superpose them, and a length of linear propagation is then necessary to overlap the pulses. In the case where the input pulses are in phase, we find that soliton formation occurs in much the same way as for the type II case, but when they are anti-phased, we find the energy scatters after collision, thus confirming that this is a phase-dependent process $[9,3]$.

Thirdly, we find that the process of soliton formation is sensitive to exactly how the input pulses overlap in transverse dimension and in time. Fig. 4 shows the transmitted energy to the output port as a function of time and transverse separation. The width of the output port was the same as that of the input pulses: four units of $y_{0}$, or $98 \mu \mathrm{m}$. The notation here is that negative transverse separation indicates that the pulses still converge at launch. The response is symmetric in time separation as $v_{\mathrm{GVM}}$ was maintained at zero.

There are four key regions in this figure. Firstly, a relatively flat plateau can be seen in the central region of the figure. This is where one crisp soliton is formed and propagates stably to the output port; it represents logic 1 output. Secondly, note the steep wall in the region of $-26 \mu \mathrm{m}$ transverse separation and zero time separation. This is where 


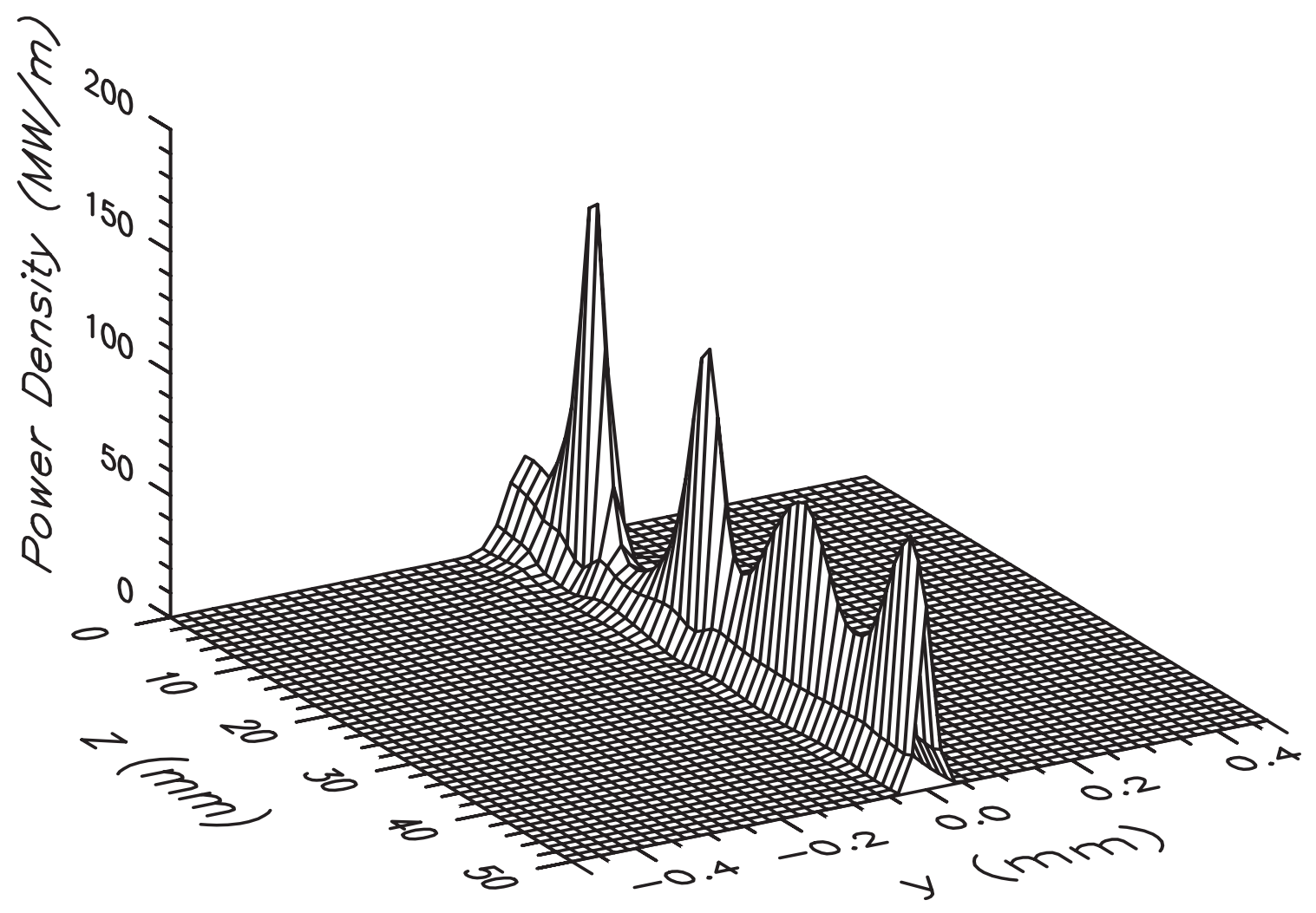

Fig. 3. Soliton formation process.

the soliton formation process becomes unstable and the initial packet of energy breaks into two solitons, which then repel each other. When the time separation is zero, they repel each other in the transverse direction; thus, they straddle the output port at the end of the switch, and no energy is transmitted. However, with the right ratio of initial transverse separation and time separation, we find that the break-up is purely along the longitudinal (time) coordinate. This results in both solitons arriving at the output port one after the other - their combined energy being visible in Fig. 4 as the "arms" leading away from the plateau: the third region. This behaviour is highly undesirable but may be easily avoided: the initial transverse separation of the pulses is determined by the geometry of the lead in channels in the switch, and as such is a manufacturing tolerance issue. If the switch can be manufactured so that this separation is within an appropriate tolerance band (say
$-8 \pm 2 \mu \mathrm{m}$ for this set-up), then all should be well. We will see a response which is primarily a function of time separation. This is shown in Fig. 5 for $-9.8 \mu \mathrm{m}$ transverse separation, and brings us to the fourth and final region of interest - the plateau wall in this vicinity.

Here, we see what appears to be a fairly digital response to the time separation of the input pulses. In fact, the switch appears to be capable of rising from a near zero response to near maximum response for about a 1 fs change in input pulse timing. The effect is not quite as digital as the wall at the front of Fig. 4 (earlier referred to as the second region), because here the break-up mechanism forms three solitons: two flying away with both transverse and longitudinal velocities, and the third remaining central. In order that the response be as digital as possible, we require that any soliton break-ups be fully effected before the soliton reaches the output port. This is why we 




Fig. 4. Transmitted energy for the type II interaction with a relative convergence angle of $0.56^{\circ}$.

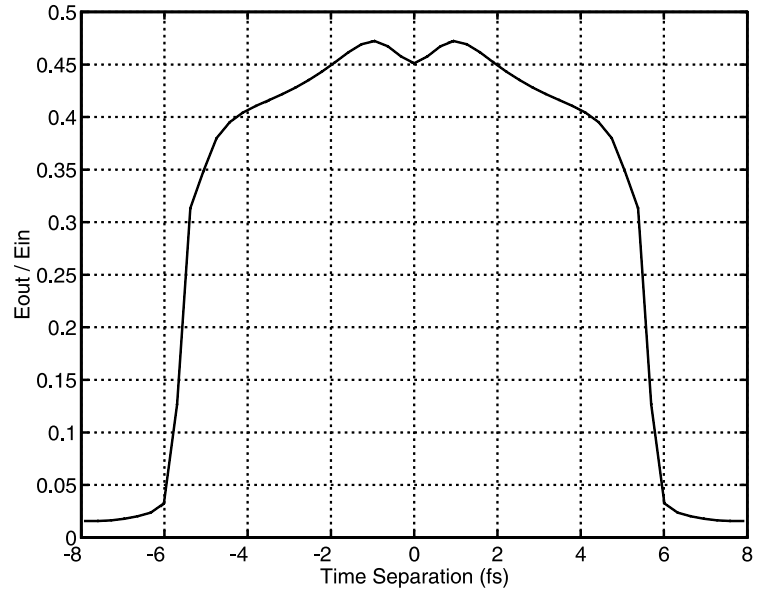

Fig. 5. Transmitted energy vs. time separation for transverse separation $=-9.8 \mu \mathrm{m}$.

required a nonlinear propagation of 10 units of $z_{0}$, as stated earlier.

Finally, we must consider the reality that there will be some degree of GVM between the fundamental fields. This has been investigated using the

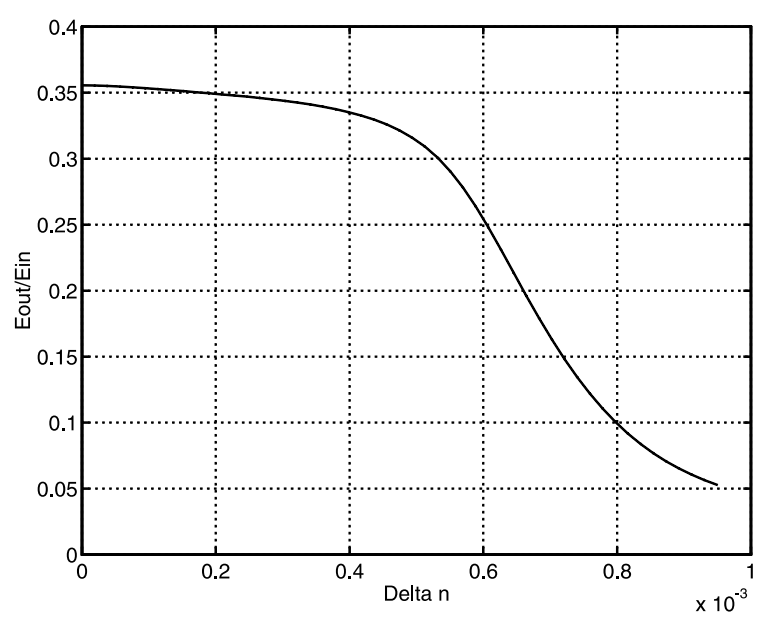

Fig. 6. Transmitted energy vs. FF GVM.

base case (as used in Fig. 3) and by varying the $\mathrm{GVM}$ from $v_{\mathrm{GVM}}=0$ to $v_{\mathrm{GVM}}=2$. The results are shown in Fig. 6, in which the abscissa is shown in units of $\left|n_{\mathrm{g} 2}-n_{\mathrm{g} 1}\right|$. Here, we can see that a mismatch of up to 0.0005 in $\left|n_{\mathrm{g} 2}-n_{\mathrm{g} 1}\right|$ has little effect, but beyond this, the soliton fails to form. 
In conclusion, we have shown that it is possible to achieve an all-optical AND gate using spatiotemporal soliton formation in a parametric medium. The interaction between fully degenerate input pulses was studied briefly and proved to be highly sensitive to the relative phase difference between the input pulses. The interaction between input pulses that were nondegenerate in polarisation was studied in detail and was found to be complex in nature. In particular, a highly digital response with regard to relative pulse timing was found. Our estimates indicate that pulse durations as short as $30 \mathrm{fs}$ are feasible, with pulse energies in the picojoule range and digital timing discrimination as short as $1 \mathrm{fs}$, in this idealised model.

\section{References}

[1] T.E. Bell, Optical computing: a field in flux, IEEE Spectrum 23 (1986) 34-57.

[2] X. Liu, K. BecKwitt, F.W. Wise, Noncollinear generation of optical spatiotemporal solitons and application to ultrafast digital logic, Phys. Rev. E 61 (2000) 4722-4725.

[3] P.D. Drummond, K.V. Kheruntsyan, H. He, Novel solitons in parametric amplifiers and atom lasers, J. Opt. B 1 (1999) 387-395.

[4] B.A. Malomed, P.D. Drummond, H. He, A. Berntson, D. Anderson, M. Lisak, Spatiotemporal solitons in multidimensional optical media with a quadratic nonlinearity, Phys. Rev. E 56 (1997) 4725-4735.

[5] X. Liu, L.J. Qian, F.W. Wise, Generation of optical spatiotemporal solitons, Phys. Rev. Lett. 82 (1999) 46314634.

[6] P.D. Drummond, Formation and stability of vee simultons, Opt. Commun. 49 (1984) 219-223.

[7] M.N. Islam, C.E. Soccolich, Billiard-ball soliton interaction gates, Opt. Lett. 16 (1991) 1490-1492.

[8] G. Leo, G. Assanto, W.E. Torruellas, Intensity-controlled interactions between vectorial spatial solitary waves in quadratic nonlinear media, Opt. Lett. 22 (1997) 7-9.

[9] G. Assanto, Transistor action through nonlinear cascading in type-II interactions, Opt. Lett. 20 (1995) 15951597.

[10] P.D. Drummond, Central partial difference algorithms, Comput. Phys. Commun. 29 (1983) 211-225.

[11] M.J. Werner, P.D. Drummond, Pulsed quadrature-phase squeezing of solitary waves in $\chi^{(2)}$ parametric waveguides, Phys. Rev. A 56 (1997) 1508-1518. 